Planetary Science and Astrobiology Decadal Survey 2023 - 2032 White Paper

\title{
The Large UV / Optical / Infrared Surveyor (LUVOIR) Telling the Story of Life in the Universe
}

\author{
Principal Author \\ Name: Aki Roberge \\ Institution: NASA Goddard Space Flight Center \\ Email: Aki.Roberge@nasa.gov \\ Phone: 301-286-2967

\section{Co-Authors}

Science and Technology Definition Team Members: Debra Fischer (Yale), Bradley Peterson (Ohio State), Jacob Bean (U of Chicago), Daniela Calzetti (U of Mass Amherst), Rebekah Dawson (Penn State), Courtney Dressing (UC - Berkeley), Lee Feinberg (NASA GSFC), Kevin France (U of Colorado - Boulder), Olivier Guyon (U of Arizona), Walter Harris (U of Arizona / LPL), Mark Marley (NASA Ames), Victoria Meadows (U of Washington), Leonidas Moustakas (JPL), John O'Meara (Keck Observatory), Ilaria Pascucci (U of Arizona / LPL), Marc Postman (STScI), Laurent Pueyo (STScl), David Redding (JPL), Jane Rigby (NASA GSFC), David Schiminovich (Columbia), Britney Schmidt (Georgia Tech), Karl Stapelfeldt (JPL), Christopher Stark (STScl), Jason Tumlinson (STScl)

International Representatives: Martin Barstow (U of Leicester, UK), Lars Buchhave (National Space Institute, Denmark), Nicolas Cowan (McGill, Canada), José Dias do Nascimento Jr. (Brazilian Federal U, Brazil), Marc Ferrari (LAM, France), Ana Gomez de Castro (U Complutense de Madrid, Spain), Kevin Heng (U of Bern, Switzerland), Thomas Henning (Max Planck, Germany), Michiel Min (Netherlands Institute for Space Research, Netherlands), Antonella Nota (STScl, ESA), Takahiro Sumi (Osaka U, Japan)

Study Office/\& Engineering Team: Giada Arney (NASA GSFC), Matthew Bolcar (NASA GSFC), Julie Crooke (NASA GSFC), Shawn Domagal-Goldman (NASA GSFC), Jason Hylan (NASA GSFC)

Many others substantially contributed to the work described in this white paper. 
The Large UV/Optical/Infrared Surveyor (LUVOIR) is a mature concept for a large multipurpose space telescope. Driving science goals for this observatory are to find dozens of potentially Earth-like exoplanets and search them for signs of habitability and life. Another key goal is to place the solar system within the general context of planetary systems by studying hundreds of diverse exoplanets and bodies throughout the solar system itself. LUVOIR represents an opportunity for partnership on a cross-disciplinary mission at an early development stage.

\section{Summary of Key Science Objectives}

Ages-old questions and investigations earned us the revelations that the stars are Suns swirling in a vast galaxy, itself one of myriads of islands in an expanding cosmos. Now we have crossed another threshold of discovery: there are planets around other stars (e.g., Mayor \& Queloz 1995). At this key point in human history, tracing a path from the dawn of the universe to life-bearing worlds is within our grasp. This monumental objective demands powerful and flexible new tools, as well as application of multi-disciplinary scientific skills.

The next frontier is to extend our characterization capabilities to rocky exoplanets and find the "pale blue dots" in the solar neighborhood. With a large enough sample size,

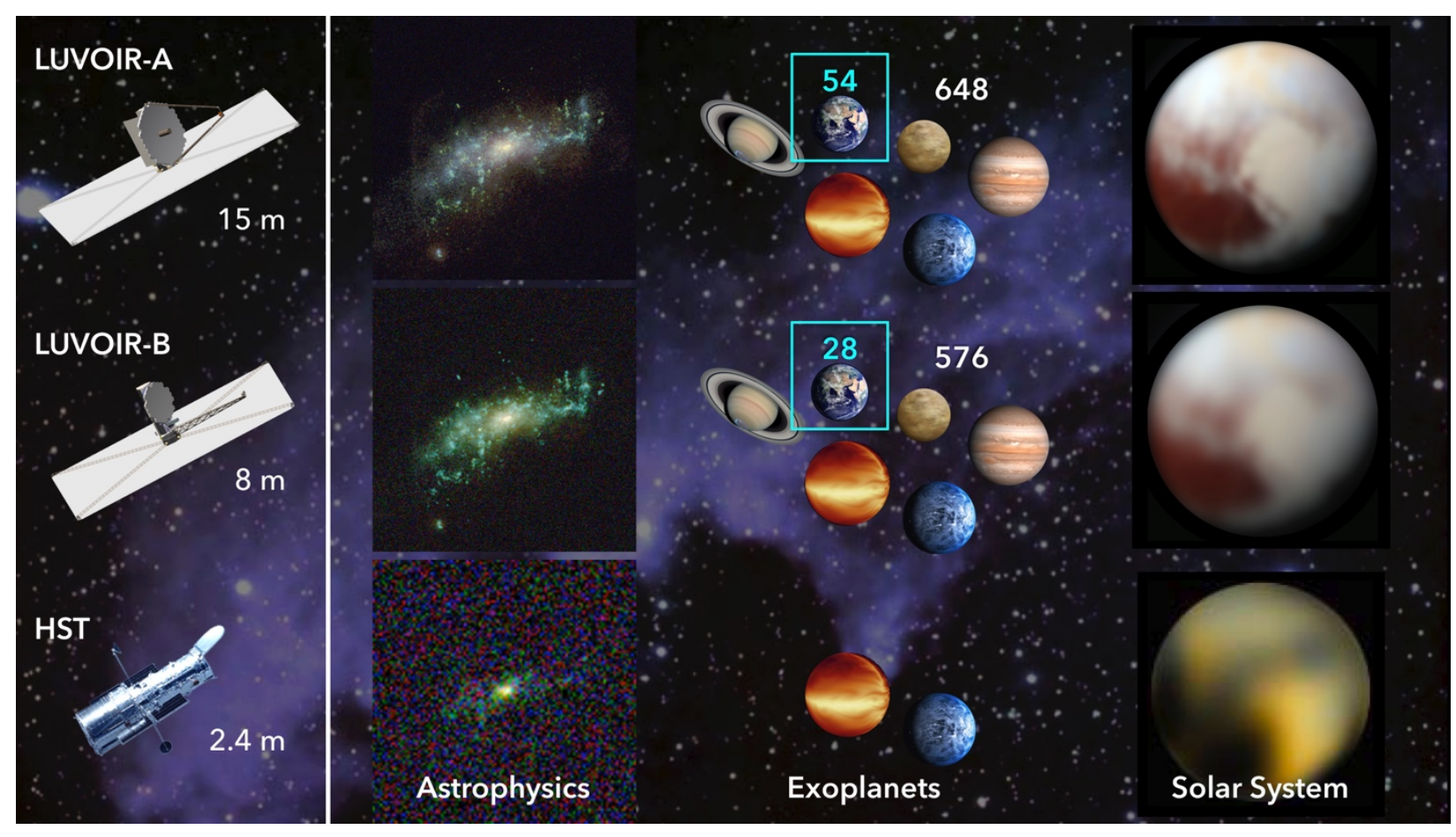

Figure 1: LUVOIR will revolutionize huge areas of space science. It will dramatically increase the sample size and diversity of exoplanets that can be studied, providing dozens of Earth-like exoplanet candidates (54 with LUVOIR-A and 28 with LUVOIR-B) and hundreds of nonhabitable exoplanets (648 with LUVOIR-A and 576 with LUVOIR-B). LUVOIR will also provide near-flyby quality observations of solar system bodies (HST Pluto image from Buie et al. 2010). Credits: NASA / New Horizons / M. Postman (STScl) / A. Roberge (NASA GSFC) 


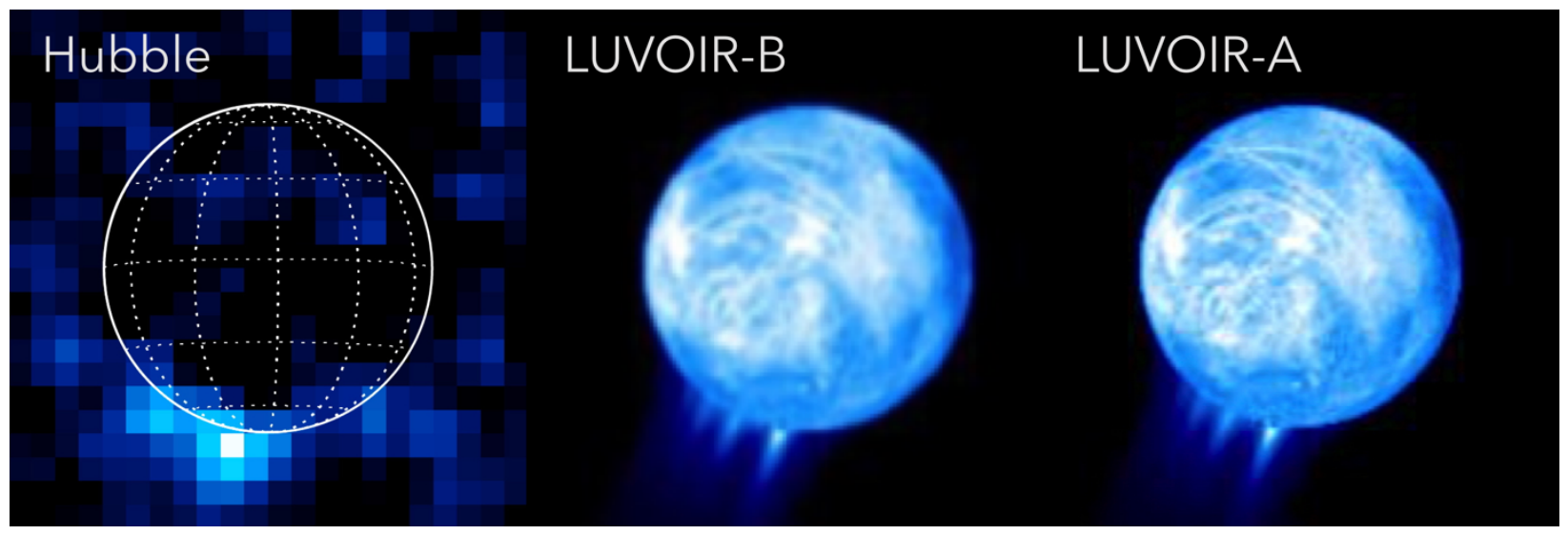

Figure 2: LUVOIR can monitor individual plumes from solar system ocean moons with flexible cadence over long timescales. The left panel shows an aurora on Europa observed with HST (Roth et al. 2014). This far-UV hydrogen emission (Lyman- $\alpha$ ) comes from dissociation of water vapor in plumes escaping through the moon's ice shell. The center and right panels show simulations of how this Lyman- $\alpha$ emission from Europa might look observed with two variants of LUVOIR (the 8-m LUVOIR-B and the 15-m LUVOIR-A). The moon's surface is bright due to reflected solar Lyman- $\alpha$ emission, which was below the background in the HST image. Credit: G. Ballester (LPL) / R. Juanola-Parramon (NASA GSFC)

scientists can determine whether habitable, Earth-like conditions are rare or common on nearby worlds and then probe them for signs of life (e.g., atmospheric oxygen). Focusing on the planetary systems most like the solar system, those with Earth-size exoplanets orbiting in the habitable zones of Sun-like stars, increases the chances of finding and recognizing atmospheric biosignatures. Concurrently, we will nurture a new discipline - comparative exoplanetology - by studying a huge range of exoplanets and comparing them with the vastly better studied solar system planets.

A vital part of establishing that context is deeper understanding of the solar system itself (see Science White paper by R. Juanola-Parramon). The bodies of the solar system, from small trans-Neptunian objects (TNOs) to giant planets, represent a treasure trove of information on the formation of the solar system, atmospheric processes, and dynamical evolution. Furthermore, the planetesimals of the outer solar system are key for deciphering the delivery of volatile and organic materials to the inner planets. Most excitingly, the icy ocean moons of the giant planets are important environments for driving a broader understanding of habitable conditions in the solar system and elsewhere (Figure 2).

There is a vital role to play for remote sensing observations of solar system bodies, allowing access to more individual objects than can be visited by spacecraft and enabling monitoring over long timescales with flexible cadence. However, to date those telescope observations have lacked the spatial resolution and sensitivity necessary to study the detailed surface features revealed by spacecraft. New concepts for future large space telescopes, developed under the aegis of astrophysics, would provide the dramatic increases needed to revolutionize solar system remote sensing. 


\section{The LUVOIR Mission Concept}

The LUVOIR mission concept is one of four Large Mission Concepts studied in preparation for the 2020 Astrophysics Decadal Survey (Astro2020). The study was initiated in Jan 2016, under the leadership of a Science and Technology Definition Team (STDT) drawn from the community. Over 3.5 years, the STDT and the LUVOIR engineering team with essential input and assistance from the broader community - worked closely to produce one of the most detailed and mature large mission concepts ever presented to an Astrophysics Decadal Survey.

The records and results of this work were submitted to NASA HQ and Astro2020 last year in the form of a Final Report ${ }^{1}$ and additional technical material (e.g., master equipment lists). Table 1 shows a summary of the two LUVOIR design variants. Solar system-specific hardware capabilities were incorporated into both variants. Note in particular the large instantaneous field of regard, which allows observations of Venus at maximum elongation and solar system bodies exterior to Earth's orbit at opposition.

The scope of science enabled by LUVOIR is truly vast, encompassing all the topics addressed by Hubble and more. The LUVOIR STDT therefore decided to focus on a set of "Signature Science Cases" (SSCs) that could be completely executed within LUVOIR's initial

Table 1: Summary of the LUVOIR space telescope concepts

\begin{tabular}{|c|c|c|}
\hline & LUVOIR-A & LUVOIR-B \\
\hline Telescope diameter & $15-m$ & $8-m$ \\
\hline Location & \multicolumn{2}{|l|}{ Earth-Sun Lagrange 2} \\
\hline Telescope properties & \multicolumn{2}{|c|}{$\begin{array}{l}\text { Diffraction limited at } 500 \mathrm{~nm} ; 270 \mathrm{~K} \text { telescope } \\
\text { operating temperature }\end{array}$} \\
\hline Lifetimes \& launch date & \multicolumn{2}{|c|}{$\begin{array}{l}\text { 5-year prime mission; } 10 \text { years of consumables; } 25 \text { - } \\
\text { year lifetime goal for non-serviceable components; } \\
\text { proposed launch date in late } 2030 \text { s }\end{array}$} \\
\hline Instantaneous field of regard & \multicolumn{2}{|c|}{ Sun-Telescope-Target angles $\geq 45^{\circ}$} \\
\hline Moving target tracking speed & \multicolumn{2}{|c|}{$\leq 60$ milliarcsec/second (2x speed of JWST) } \\
\hline $\begin{array}{l}\text { Total wavelength range of } \\
\text { candidate instrument suite }\end{array}$ & \multicolumn{2}{|l|}{$100 \mathrm{~nm}-2500 \mathrm{~nm}$} \\
\hline \multirow[t]{4}{*}{$\begin{array}{l}\text { Current candidate instrument } \\
\text { capabilities }\end{array}$} & \multicolumn{2}{|c|}{$\begin{array}{l}\text { High-contrast near-UV/optical/ NIR imaging \& low- } \\
\text { resolution spatially resolved spectroscopy }\end{array}$} \\
\hline & \multicolumn{2}{|c|}{ Wide-field near-UV/optical/NIR imaging } \\
\hline & \multicolumn{2}{|c|}{ UV/optical multi-resolution, multi-object spectroscopy } \\
\hline & UV spectropolarimetry & $\ldots$ \\
\hline \multicolumn{3}{|l|}{ Max spatial resolution at $1 \mu \mathrm{m}$} \\
\hline Venus, Jupiter, Saturn & $15 \mathrm{~km}, 50 \mathrm{~km}, 102 \mathrm{~km}$ & $28 \mathrm{~km}, 94 \mathrm{~km}, 193 \mathrm{~km}$ \\
\hline Uranus, Neptune, $40 \mathrm{AU}$ & $217 \mathrm{~km}, 346 \mathrm{~km}, 464 \mathrm{~km}$ & 409 km, 653 km, 875 km \\
\hline
\end{tabular}

${ }^{1}$ Available at https://www.luvoirtelescope.org/ 


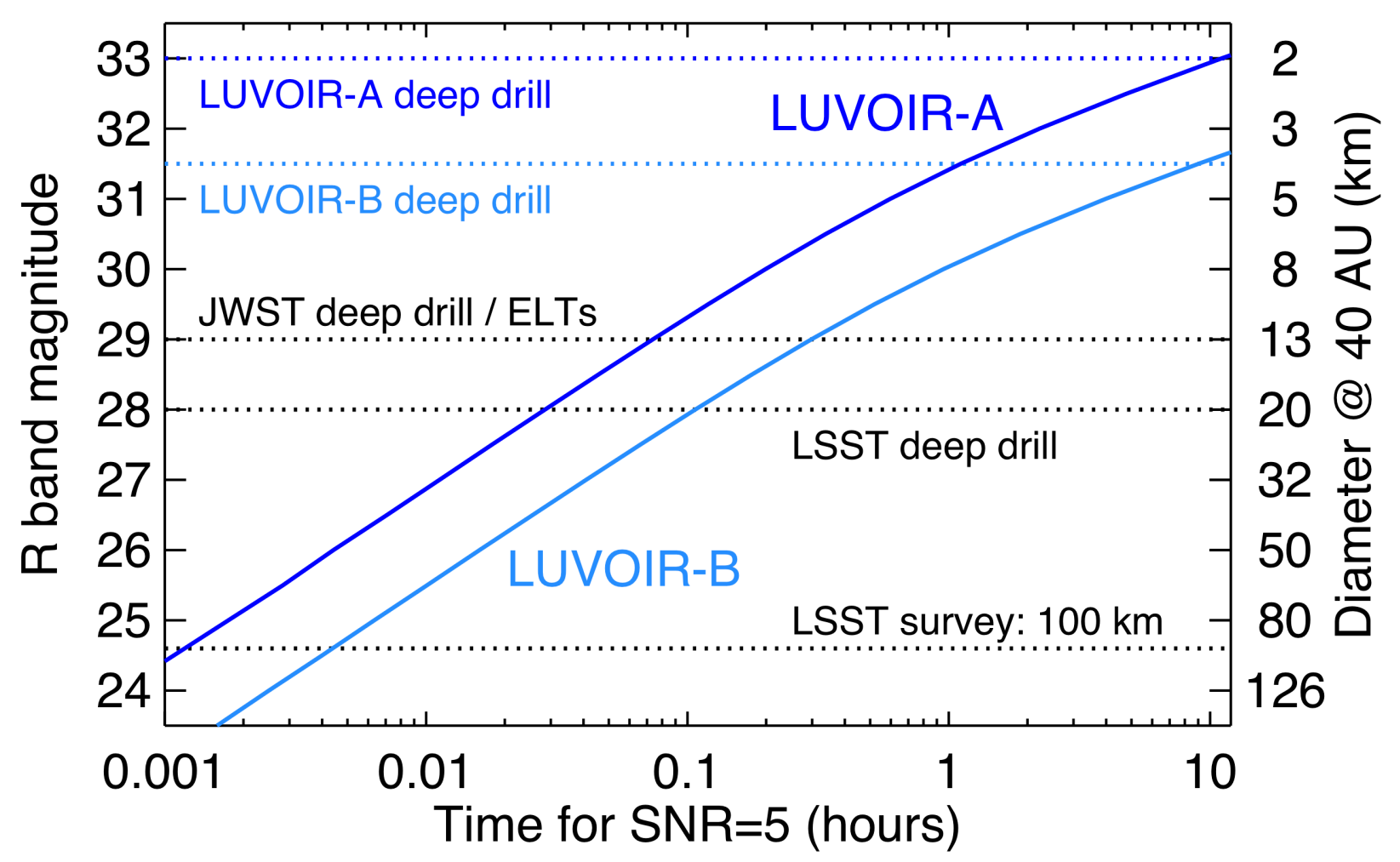

Figure 3: LUVOIR can reach down to smaller trans-Neptunian objects (TNOs) than any other current or planned facility, including the James Webb Space Telescope (JWST), the 30-meterclass ground-based Extremely Large Telescopes (ELTs), and the Rubin Observatory Legacy Survey of Space and Time (LSST). The solid curves show the R band apparent magnitude of TNOs detectable at SNR $\sim 5$ as a function of exposure time for LUVOIR-A (dark blue) and LUVOIR-B (light blue). The right $y$-axis shows the sizes of TNOs at $40 \mathrm{AU}$ corresponding to the $\mathrm{R}$ mags. These assume a single image that is depth-optimized to the Classical KuiperEdgeworth Belt by moving target tracking. Horizontal dotted lines show limits for other TNO surveys. Credit: R. Dawson (PSU) / A. Roberge (NASA GSFC)

5 -year prime mission. Two of the twelve SSCs center on solar system observations - SSC \#3: The search for life in the solar system and SSC\#6: Small bodies in the solar system. The former is a spectral imaging campaign to monitor far-UV plume emission from Europa (Figure 2), as well as multi-epoch optical/near-IR imaging of 6 additional ocean moons. The latter is a deep optical/NIR survey to detect up to 100 very small TNOs (Figure 3) and measure the orbits of binary TNOs.

The Signature Science Cases represent some of the most compelling observing programs that scientists might do with LUVOIR at the limits of its performance. As compelling as they are, they should not be taken as a complete specification of LUVOIR's future scientific potential. The SSCs take a total of 3.5 years to execute with LUVOIR-A and 4.2 years with LUVOIR-B, leaving time for additional science even within the 5-year prime mission and staggering opportunities within the 10-year consumables lifetime. We fully expect that the creativity of the community, empowered by the revolutionary capabilities of LUVOIR, will 
ask questions, acquire data, and solve problems beyond those discussed in the LUVOIR Final Report.

\section{Technical Overview}

The LUVOIR Team developed two distinct observatory concepts: the 15-m LUVOIR-A (Figure 4), designed for launch in the SLS Block 2 vehicle; and the 8-m LUVOIR-B (Figure 5), designed to fit in heavy-lift launch vehicles with 5-m fairings similar to those in use today. By studying two designs, we gain better understanding of a complex trade space, reveal how science return scales with different technical choices, and establish robustness to uncertainties such as future launch vehicle capabilities and budget constraints. It is important to recognize that LUVOIR-A and -B represent proof-of-concept point designs within a family of UV/optical/NIR observatories, demonstrating feasibility and providing information for the future. LUVOIR's main features are:

- Scalable, serviceable architecture for an observatory at Earth-Sun L2

- A 5-year prime mission, with 10 years of on-board consumables. Non-servicable components have a 25-year lifetime goal

- Large, segmented, deployable telescopes designed for launch in next-generation heavy lift vehicles with large fairings (e.g., NASA's SLS Block 2, NASA's SLS Block 1B Cargo, Blue Origin's New Glenn, and SpaceX's Starship)

- UV-capable telescopes that are compatible with high-contrast exoplanet observations. Total wavelength range of the studied instrument suite is 100-2500 nm

- Telescope optical designs to alleviate polarization effects that can degrade coronagraph contrast

- A sunshade that is larger but simpler than the JWST sunshield (three layers instead of JWST's five, greatly relaxed requirements on layer positioning after deployment)

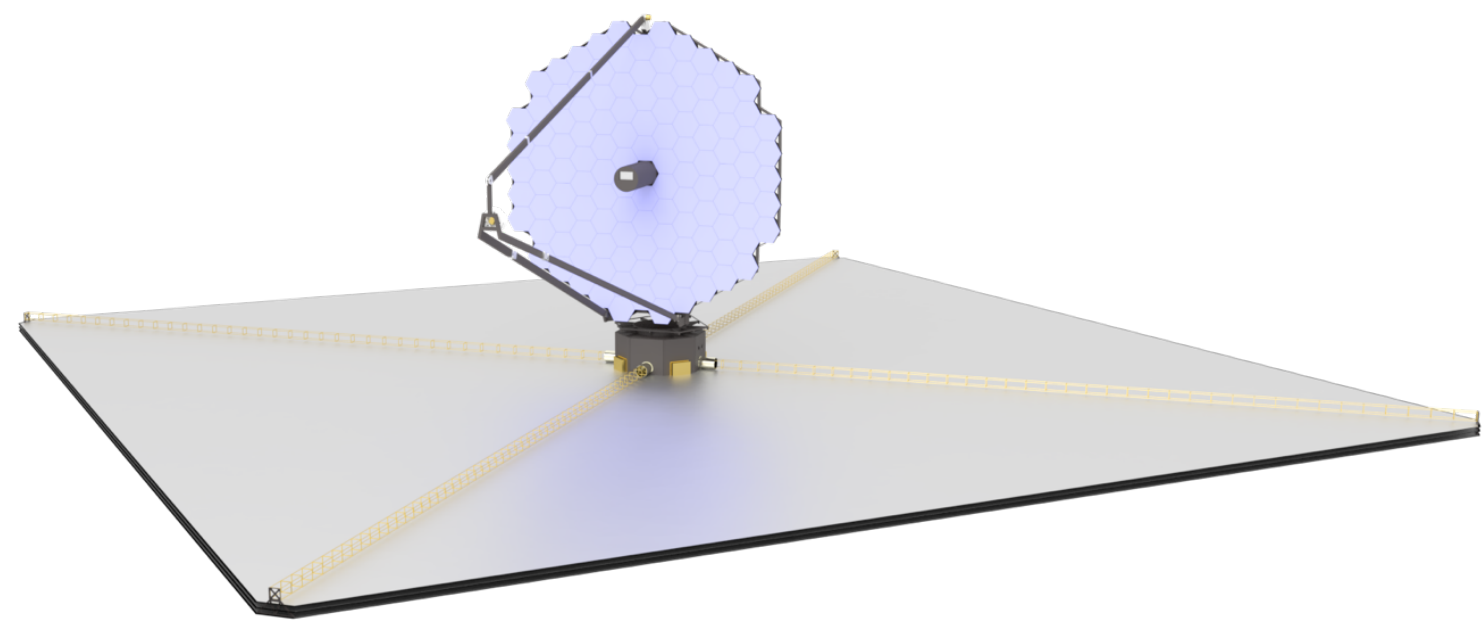

Figure 4: The LUVOIR-A observatory, with a 15-m diameter on-axis primary mirror and four instruments. Deployment and pointing animation at https://asd.gsfc.nasa.gov/luvoir/design/. Credit: A. Jones (NASA GSFC) 


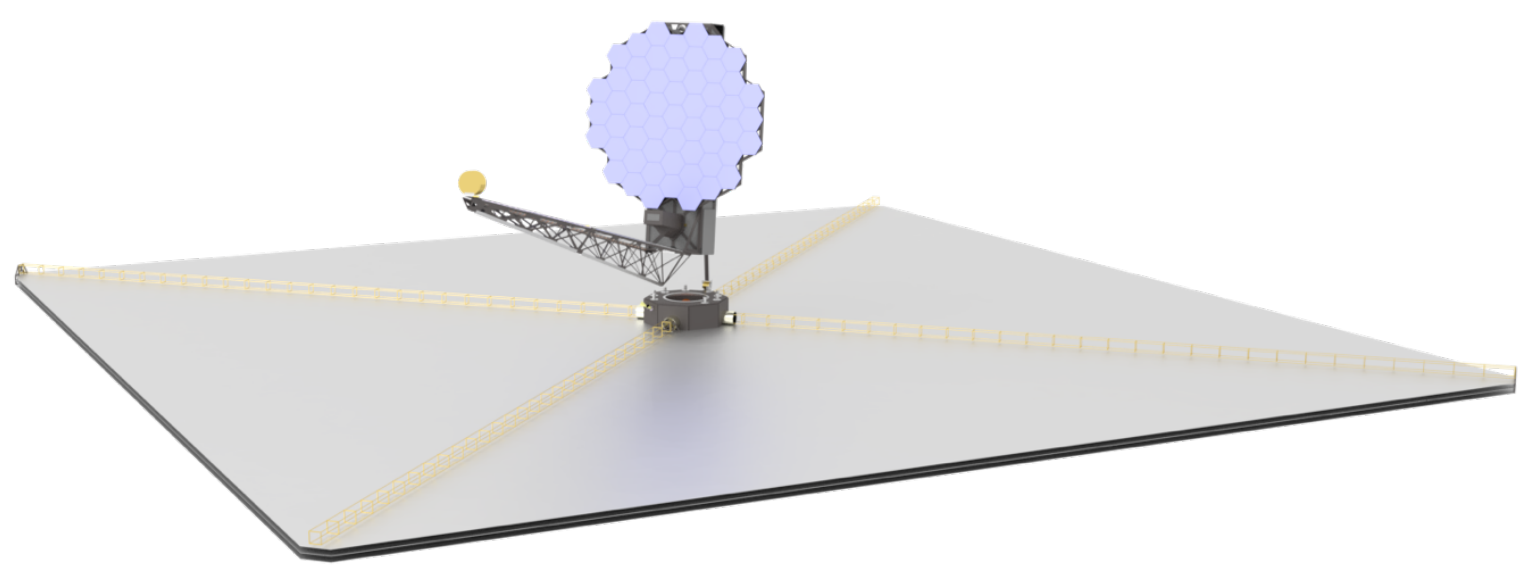

Figure 5: The LUVOIR-B observatory, with an 8-m diameter off-axis primary mirror and three instruments. Deployment and pointing animation at https://asd.gsfc.nasa.gov/luvoir/design/. Credit: A. Jones (NASA GSFC)

- An instantaneous field-of-regard that covers most of the sky (LUVOIR can point to within $45^{\circ}$ of the Sun), greatly enhancing target of opportunity, exoplanet, and solar system observations

- Moving target capability with max. tracking speed of 60 mas/sec (2x faster than JWST)

- ECLIPS: A high-contrast coronagraph with imaging cameras and integral field spectrographs spanning 200-2000 nm, capable of directly observing a wide range of exoplanets and obtaining spectra of their atmospheres

- HDI: A near-UV to near-IR imager covering 200-2500 nm, diffraction limited and Nyquist sampled at $500 \mathrm{~nm}$, with high precision astrometry capability

- LUMOS: A far-UV imager and multi-resolution, multi-object spectrograph covering 100-1000 nm, capable of simultaneous observations of up to hundreds of sources

- POLLUX: A high-resolution, point-source UV spectropolarimeter covering 100-400 nm, designed for LUVOIR-A. This instrument study was contributed by a consortium of European institutions, with support from the French Space Agency

A transformative facility like LUVOIR with its large aperture and need for high-contrast observations of Earth-like exoplanets requires new approaches to designing and realizing an observatory. Far more than previous space telescopes, LUVOIR must be considered as an integrated system and was designed for adaptability:

- Scaleable architecture to leverage a rapidly changing launch vehicle landscape

- Modular design to ease on-orbit servicing and integration \& testing on the ground

- Several layers of active wavefront control in the telescopes and within the coronagraphs to relax constraints on hardware precision and on-the-ground verification

- Minimization and isolation of vibrations and thermal disturbances throughout the observatory 
Table 2: Key material in the LUVOIR Final Report at https://www.luvoirtelescope.org

\begin{tabular}{|c|c|c|}
\hline & $\begin{array}{l}\text { Final Report } \\
\text { Location }\end{array}$ & Description / Comments \\
\hline Final Report Summary & Chapter 1 & 64-pg. summary of 426-pg. report \\
\hline Science Goals \& Requirements & Chapters $3-6$ & Additional science cases in Appendix A \\
\hline Design Reference Missons & Appendix B & Detailed observing plans \\
\hline Science Mission Traceability & $\begin{array}{l}\text { Chapter } 7 \& \\
\text { Appendix C }\end{array}$ & \\
\hline Observatory Technical Design & Chapter 8 & Telescope, instruments, \& spacecraft \\
\hline $\begin{array}{l}\text { Mission Operations \& Ground } \\
\text { Segment }\end{array}$ & Chapter 9 & \\
\hline Launch Vehicles & Chapter 10 & \\
\hline Mission Cost Estimates & $\begin{array}{l}\text { Section } 1.13 \& \\
\text { Appendix J }\end{array}$ & $50-70 \%$ confidence cost ranges given \\
\hline Technology Development Plan & Chapter 11 & $\begin{array}{l}\text { TRL assessments, development } \\
\text { schedule, \& estimated total cost of plan }\end{array}$ \\
\hline $\begin{array}{l}\text { Project Management \& } \\
\text { Systems Engineering }\end{array}$ & Chapter 12 & \\
\hline $\begin{array}{l}\text { Mission Development } \\
\text { Schedules }\end{array}$ & $\begin{array}{l}\text { Section } 12.6 \& \\
\text { Appendix } G\end{array}$ & Assume Phase A start on Jan 1, 20205 \\
\hline
\end{tabular}

\section{Additional Information}

Much more scientific and technical information about LUVOIR is available in the Final Report and Appendices. In Table 2, we identify where to find key information.

\section{Conclusion}

In sum, LUVOIR is an ambitious mission concept that will provide the most science for the most scientists, but will require careful attention and new ways of executing large missions to make it feasible in terms of cost and schedule. However, the LUVOIR Team believes that this can be done, and has worked to develop plans to effectively realize our vision for a revolutionary observatory while maintaining a balanced portfolio of NASA science missions. We further believe that LUVOIR's compelling science goals will excite the scientific community, the public, and the stakeholders in government, conceivably leading to sustained and increased support for space science in the US.

\section{References}

Buie, M. W., Grundy, W. M, Young, E. F., et al. (2010). AJ, 139, 1128

Juanola-Parramon, R., et al. (2020). "Solar System Science with Space Telescopes",

Planetary Science \& Astrobiology Decadal Survey Science White Paper

LUVOIR Final Report (2019). https://arxiv.org/abs/1912.06219

Mayor, M. \& Queloz, D. (1995). Nature, 378, 355 